\title{
Lane Detection Algorithm Based on Road Structure and Extended Kalman Filter
}

\author{
Jinsheng Xiao, School of Electronic Information, Wuhan University, Wuhan, China \\ Wenxin Xiong, School of Electronic Information, Wuhan University, Wuhan, China \\ Yuan Yao, College of Physical Science and Technology, Central China Normal University, Wuhan, China \\ Liang Li, School of Electronic Information, Wuhan University, Wuhan, China \\ Reinhard Klette, School of Engineering, Computer and Mathematical Sciences, Auckland University of Technology, \\ Auckland, New Zealand
}

(iD) https://orcid.org/0000-0001-8818-7145

\begin{abstract}
Lane detection still demonstrates low accuracy and missing robustness when recorded markings are interrupted by strong light or shadows or missing marking. This article proposes a new algorithm using a model of road structure and an extended Kalman filter. The region of interest is set according to the vanishing point. First, an edge-detection operator is used to scan horizontal pixels and calculate edge-strength values. The corresponding straight line is detected by line parameters voted by edge points. From the edge points and lane mark candidates extracted above, and other constraints, these points are treated as the potential lane boundary. Finally, the lane parameters are estimated using the coordinates of the lane boundary points. They are updated by an extended Kalman filter to ensure the stability and robustness. Results indicate that the proposed algorithm is robust for challenging road scenes with low computational complexity.
\end{abstract}

\section{KEYWORDS}

Extended Kalman Filter, Lane Detection, Road Models, Vanishing Point

\section{INTRODUCTION}

Advanced driver assistance systems are gradually being incorporated into vehicles. They can either alert the driver in dangerous situations or take an active part in the driving. They are expected to become more complex towards full autonomy during the next decade. One of the main bottlenecks in the development of such systems is the perception problem. Road, lane and obstacle detection (Huang et al., 2009; Hillel et al., 2014) are important vision perception problems. In this paper lane detection is considered. The main perceptual cues for human driving include road color and texture, road boundaries, and lane markings. Autonomous vehicles are expected to share the road with human drivers. It is unrealistic to expect the huge investments to construct and maintain special infrastructure only for autonomous vehicles. Autonomous vehicles will therefore most likely continue to rely on the same perceptual cues that human drivers do (Aly, 2008; Xiao et al., 2015).

The road and lane detection generally includes the following five modules. They are image preprocessing, feature extraction, road or lane model fitting, temporal integration, and image-to-world correspondence (Meng et al., 2010; Xu et al., 2011). A lane departure warning system was proposed

This article, originally published under IGI Global's copyright on April 1, 2020 will proceed with publication as an Open Access article starting on January 27, 2021 in the gold Open Access journal, International Journal of Digital Crime and Forensics (converted to gold Open Access January 1, 2021), and will be distributed under the terms of the Creative Commons Attribution License (http://creativecommons.org/ licenses/by/4.0/) which permits unrestricted use, distribution, and production in any medium, provided the author of the original work and original publication source are properly credited. 
by Lee (2002) to estimate the subsequent direction of lane through an edge distribution function and direction changes of vehicle movement. It failed on roads with curved and dashed lanes. Wang et al. (2004) provided an initial position for a B-snake model, then the lane detection problem can be changed into the problem of control points to determine a spline curve following a road model. Mechat et al. (2013) detected the lane using a support vector machine (SVM) based method. In this method, the model of the lane was defined by a Catmull-Rom curve, and the standard Kalman filter was adopted to estimate and track the parameters of control points. Shin et al. (2015) extended a particle-filterbased approach for lane detection, also addressing challenging road situations. Kortli et al. (2017) proposed a method based on Gauss filter and Canny edge detector to extract lane boundaries based on color information. Although this system worked properly on a lot of different conditions, there were still some problems for blur lane marks and complex road surface. Lee et al. (2017) proposed a real-time lane detection algorithm using a simple filter and Kalman filter that can be implemented in an embedded system. Even though this method was invariant against various illumination changes, it was still difficult to handle several extreme conditions such as strong light reflection, blur lane marks, low sun angle situations and lane cracks. Pan et al. (2017) proposed a dual-stage detecting strategy, which consisted of a fast Hough transform based road detection method and a reliable vanishing point-based method. However, an evaluation method was needed to judge the detection result of two stages. The lane detection modules currently provide stable results in general, but their performance under special conditions is still a research topic; those conditions might be defined by strong sunlight, hard to identify lanes, shadows caused by trees or other objects, sidewalks, zebra crossings, or text logos on the road.

In view of this, a lane detection algorithm is proposed by combining a road structure model with an extended Kalman filter. Considering the characteristics of the lane and according to the roadway geometry, a new lane model is proposed which enhances the stability and anti-jamming of the lane-detection system. The parameter space is defined to accommodate the algorithm for the lane model. Due to algorithmic developments in the area of Hough transforms (Xu et al., 2015), there is good progress to improve the processing speed. The extended Kalman filter is used to estimate and track the lane, which is the major factor for improved accuracy in the proposed lane detection. The effectiveness and robustness of the algorithm is demonstrated in this paper.

The structure of this paper is as follows. Section 2 reports about the used road model. Section 3 describes the lane detection algorithm based on road structure and extended Kalman filter. Section 4 informs about the experiments and the performed evaluation. Section 5 concludes.

\section{THE PROCESSING OF BASIC LAYER}

The estimated parameters of the lane detection in this paper are related to the lane shape and the posture of the driving vehicle. These parameters involve lane width, the shifting of vehicle, pitch angle, horizontal angle and lane curvature, etc. Figure 1 shows the flowchart of the proposed lane detection algorithm. The parameter estimation is divided into two parts. The first part is the posture of the camera and the road environment information obtained from the in-vehicle camera. The second part estimates the parameters of the road using the road lane information.

The flowchart of the algorithm in this paper begins with reading a frame of image. The adaptive edge detection operator based on the scan line is used to extract the image edge points from the $\mathrm{R}$ channel of the image. The region of interest of the algorithm is selected. The image pixel coordinate system is mapped to the road plane coordinate system. The edge points are voted in custom parameter space to get candidate lines. The geometrical characteristics and spatial continuity of the lane are collected. The non-lane lines can be excluded using lane information in the image pixel coordinate system and lane road plane coordinate system. The inner-boundary points and extended Kalman filter are combined to estimate and update lane model parameters. 


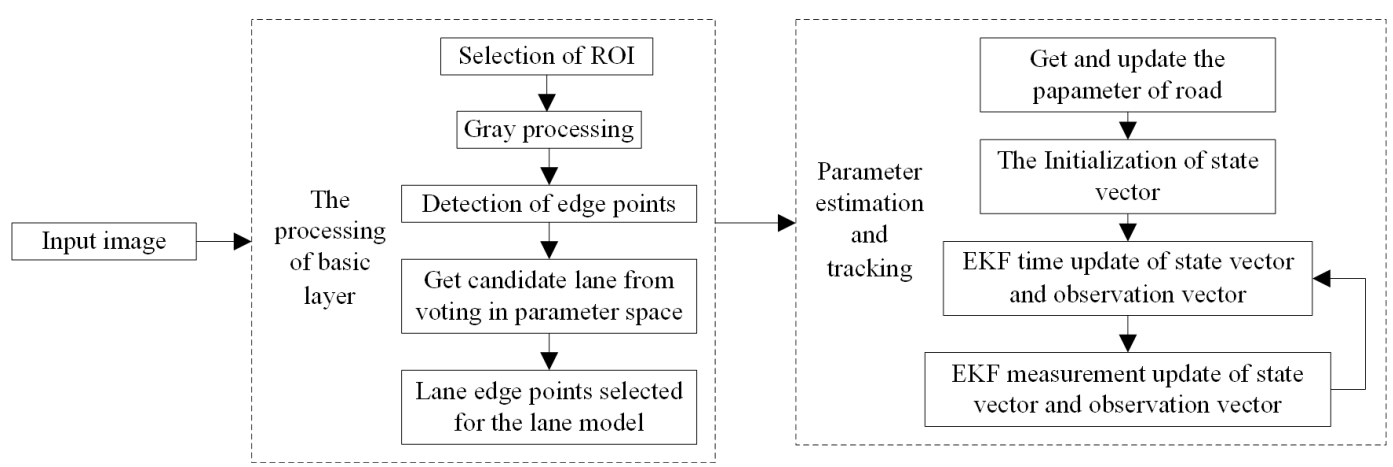

\subsection{The Region of Interest}

In order to exclude the interference information in the original input image, the proposed algorithm focuses on the image in front of the vehicle and on both sides of a certain spatial extent. This greatly reduces the image processing region to facilitate the follow-up operation of the lane detection and extraction.

Images produced by normal cameras have a perspective effect. The parallel lines that are not parallel to the projection plane will intersect in the image to a point, which is called the vanishing point (Xu et al., 2015) (see Figure 2). In the real world, the two lane lines that are parallel to each other intersect at vanishing point after perspective transformation (McDonald et al., 2001), which may be inside or outside the image (Xu et al., 2015). The region of interest is selected based on the coordinates of the vanishing point of the image in the proposed method.

The coordinates of vanishing point in the world coordinate system are:

$\mathrm{WC}=[\sin \xi / \cos \psi, \cos \xi / \cos \psi, 0]$

where $\xi$ and $\psi$ are the pitch angle and tilt angle. In this way, the calculation formula for the vanishing point matrix (Aly, 2008; Kazui et al., 2010) is as follows:

$V P=F \times P \times S \times W C$

where $\mathrm{P}$ is the rotation matrix of camera pitch angle, $\mathrm{S}$ is the rotation matrix of camera horizontal tilt angle, $\mathrm{F}$ is the offset matrix of camera focal length:

$$
P=\left[\begin{array}{ccc}
1 & 0 & 0 \\
0 & \cos \xi & \sin \xi \\
0 & -\sin \xi & \cos \xi
\end{array}\right], S=\left[\begin{array}{ccc}
\cos \psi & \sin \psi & 0 \\
-\sin \psi & \cos \psi & 0 \\
0 & 0 & 1
\end{array}\right], F=\left[\begin{array}{ccc}
f_{x} & 0 & c_{x} \\
0 & f_{y} & c_{y} \\
0 & 0 & 1
\end{array}\right]
$$

Focal length $f_{x}, f_{y}$ are the product of the physical focal length of the lens and the size of each cell in the image. $c_{x}$ and $c_{y}$ are the optical center offset of the camera.

After determining the coordinates of the vanishing point, it is necessary to reduce the computation of the processed image and interference information. The region of interest (ROI) for the image can be 
Figure 2. The vanishing point

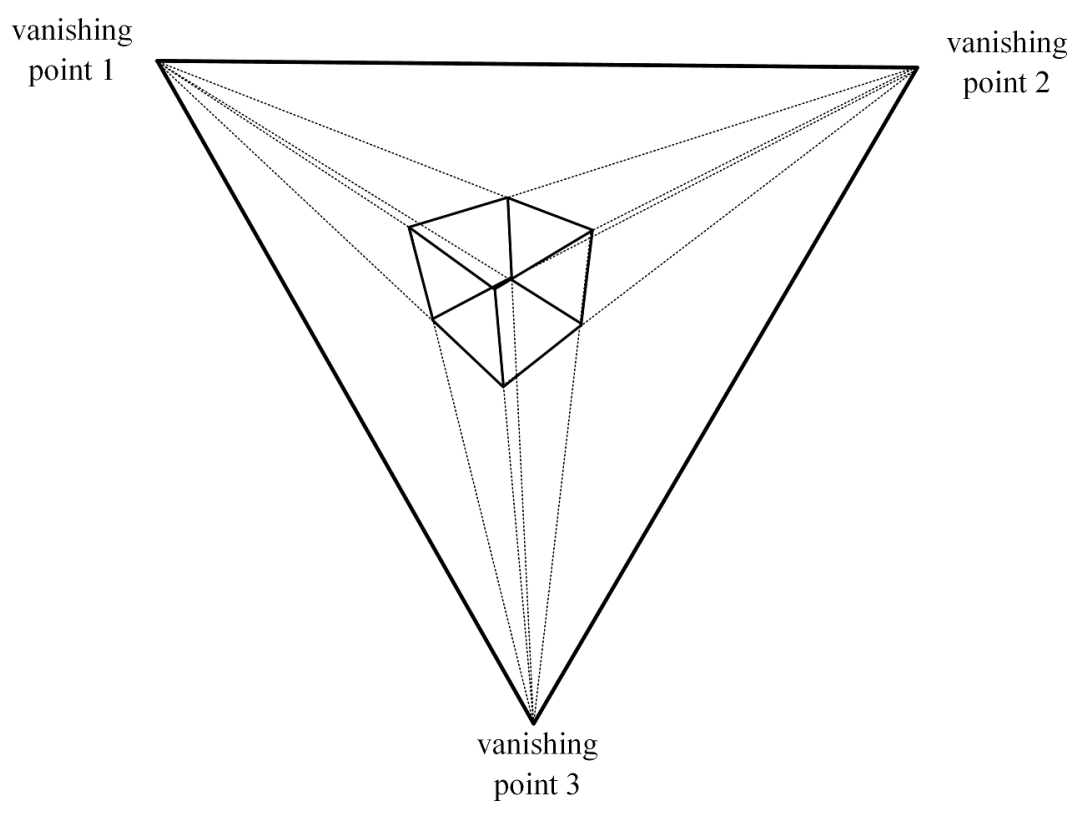

obtained, according to the ordinate of the vanishing point and the relevant parameters of the camera. Image only in the ROI is used in the process of image processing and transformation. Figure 3 is the region of interest in the proposed method.

\subsection{Gray Processing}

Considering the different visual effects of input RGB image, the following conversion formula is used to get the gray level:

$$
\text { Grayscale }=(R \cdot 0.299)+(G \cdot 0.587)+(B \cdot 0.114)
$$

The sum of R, G, B three channels' coefficient is 1 .

The lane line is the only locked target in the lane departure detection process. The color of lane is yellow and white, which is different from the road. Therefore, the Equation (4) will be used to reduce the computational burden and improve the detection efficiency.

Since the conversion formula in Equation (4) is a float operation. In the proposed algorithm, it is modified to release more hardware resources and reduce the amount of computation as following:

$$
\text { Grayscale }=(R+G+B) / 3
$$

Although the quality of the image is changed by the transformation formula, it still preserves the edge features of the target lane line in the image. This new formula significantly reduces the amount of algorithmic computation. 


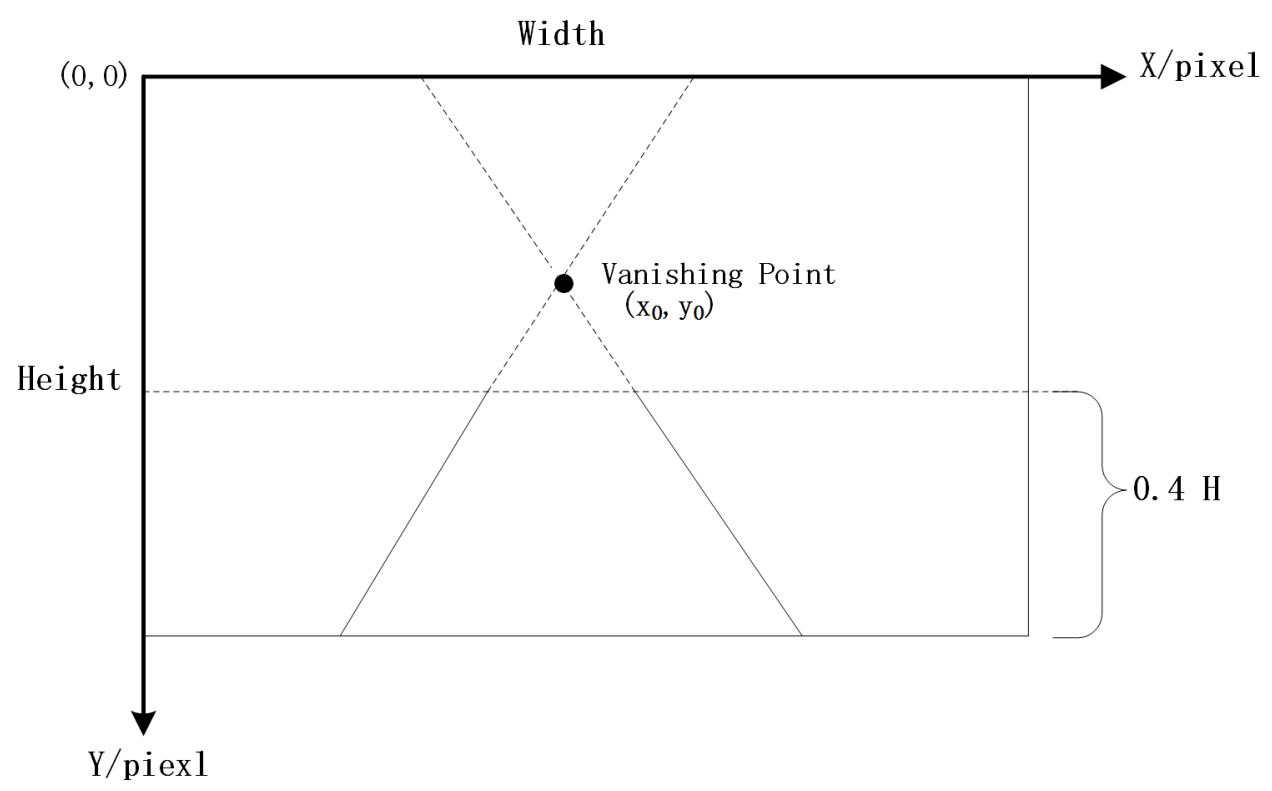

\subsection{Detection of Edge Points}

Due to the perspective effect of the camera, visible information differs for different ranges in the image of the road plane. We divide the regions of interest into several areas. A preset area is selected according to the image coordinates which are converted using the vanishing point coordinates and the real coordinates.

The customized edge-detection operator is based on scan-line processing for reducing the amount of computations. An image row forms a scanning line. If the number of calculated scan lines is greater than the maximum value, the scan lines are set under the real-world coordinates with the same distance of each line. These lines are translated into the image pixel coordinate system. A set of scan lines is shown in Figure 4, left.

By scanning through each pixel of the selected scan line, the edge strength of each pixel is calculated by the following equation. $E(i)$ is an edge strength of pixel in the scanning line. $I(i)$ represents the values of the i-th pixel. $L$ is the scale of pixel:

$$
E(i)=-\sum_{k=-L}^{-1} I(i+k)+\sum_{k=1}^{L} I(i+k)
$$

This method is different with the method which uses the same scale to compute all the pixels. The pixel scale $L$ of the adaptive edge detection operator changes based on the position of the pixels in the image. The perspective effect of the lane in the road image is considered. The length that each pixel denoting in world coordinates on the scanning lines is computed after coordinate conversion. The width of the lane is used to be divided by the proportion for each scan lines. Then the pixels used to compute the edge strength can be gotten. The pixels which have the maximum or the minimum value of edge strength are edge points. The custom edge detection operators can greatly reduce the processing time. Edge detection results are shown in Figure 4, right. It can be seen that the operator can detect the edge points of all lanes with very good accuracy and efficiency. 


\subsection{Get the Candidate Lane From Voting in Parameter Space}

After edge points are detected, they need to be aggregated into a candidate lane. The amount of computations for the traditional Hough transform (Hough, 1962) is large but can be reduced (Xu et al., 2015). Aiming at the lane model, the parameter space is defined conveniently for our algorithm. The edge points are voting in the parameter space. A diagram of the customized parameter space is shown in Figure 5. The parameter space is spanned by two parameters $p$ and $q$. The lane in the region of interest is defined by $x=p+y(q / d)$. Parameter $p$ represents the position of the line on the $x$ axis; parameter $q$ identifies the slope, i.e. the lateral position. The flowchart of our voting algorithm includes three parts:

1. The coordinate of edge points are transformed. The edge points obtained from the edge detection are in the image pixel coordinate system. The edge points need to be converted to the world coordinate system, in which the perspective effect is removed;

2. All the edge points in the selected area are traversed by parameter $q$. The corresponding $p$ was obtained through the look-up table. The accumulator increases 1. $q$ is translated from the tilt angle of the lanes. $q$ is discretized and the range of value is $\left[-88^{\circ}, 88^{\circ}\right] . p$ is discretized and the range of value is $[-5 \mathrm{~m}, 5 \mathrm{~m}]$ in the world coordinate system;

3. Different $p, q$ denote different lines. From the mapping relationship of the parameter space, the larger the value of the accumulator of $p, q$ is, the more edge points there are in the lane which are represented by this parameter. All possible candidate lines are identified by searching for the maximum value.

The wrong candidate lines are parallel to the right lane boundary in many cases. After searching for all local maximum value, it needs to check whether any pair of candidate line is crossed. If a pair of candidate lines is crossed, the line with a smaller value in the parameter space will be discarded. The judgment for determining cross-line is as follows:

$\left(p_{A}-p_{B}\right) \cdot\left(p_{B}-p_{A}+q_{B}-q_{A}\right) \geq 0$

The candidate lines can be gotten by voting algorithm in the parameter space. The geometrical characteristics and spatial continuity of the lane are still needed to exclude the unwanted points and unwanted lanes. The dual information of lane in the image pixel coordinate system and the lane road

Figure 4. Left: A set of scan lines. Right: Examples of detected edge points; input image is as shown on the left.
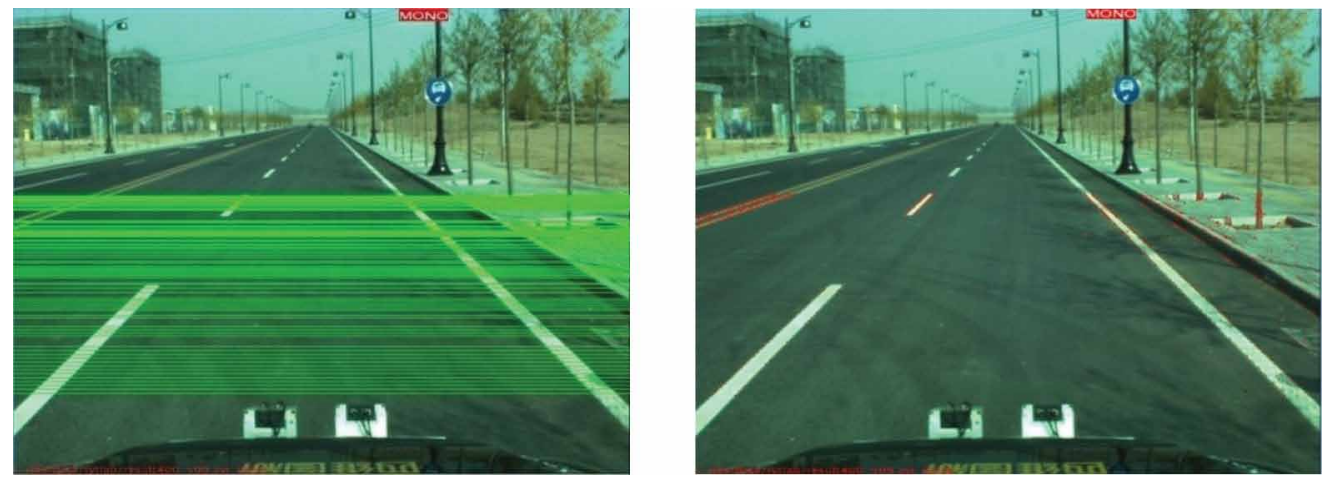


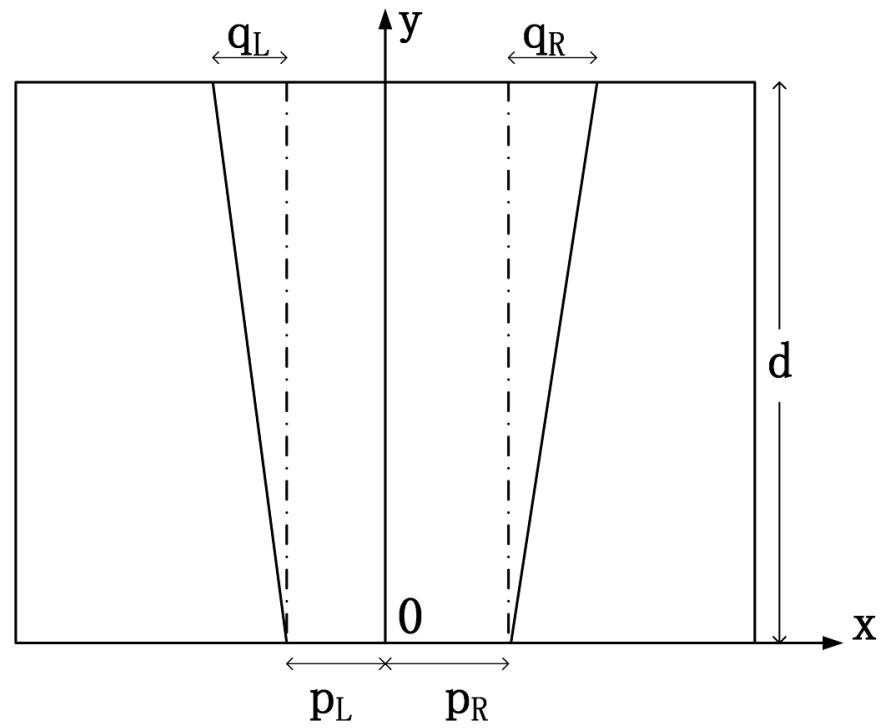

plane coordinate system is useful too. The final candidate lanes are shown in Figure 6, left, which detect the current two lanes accurately.

The lane edge points need to be selected for the lane model after the above steps. All the points should be selected, but only ten points are selected if the total number of edge points is greater than ten. The ten points are selected by same distances. The selected points are shown in Figure 6, right. The perspective effect in the road line image should be considered in the selection of boundary points which can ensure the reasonableness and accuracy of the lane tracking and estimating in the later.

Beside the single solid line, the dashed line and double line are detected in the proposed algorithm. The boundary points of solid line are continuous, while there will be a greater distance between the boundary points in the dashed line. The difference of two adjacent boundary points is calculated. The averages of the differences are used to discriminate the solid or dashed.

Figure 6. Left: Candidates of lane boundary lines. Right: Selected boundary points.
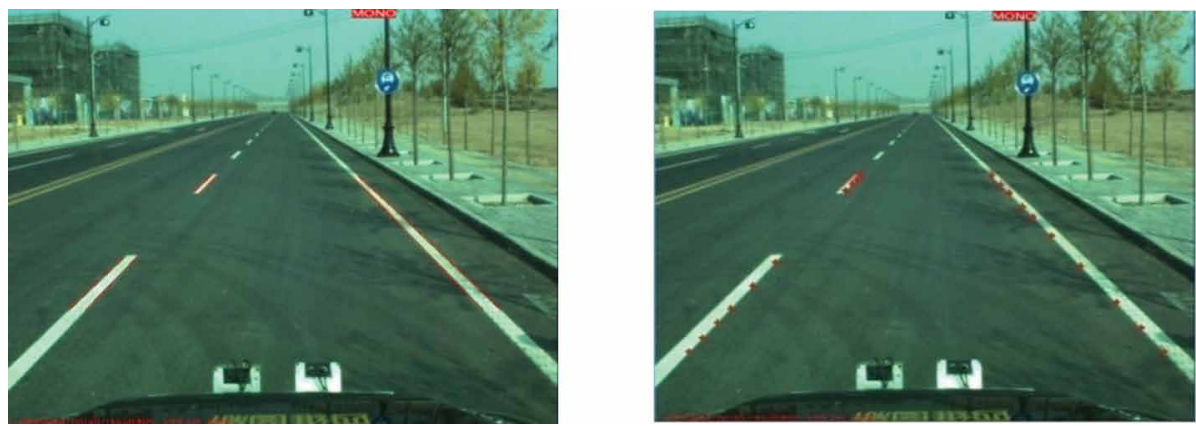


\section{LANE DETECTION COMBINED OF LANE MODEL AND EXTENDED KALMAN FILTER}

Vehicle shaking, light changing and vehicle interference, all can lead to the jitter of the collected images. One or several frames can't identify the lane lines due to the reasons of broken lane, dirty road, shadows, and so on. The filter algorithm is used to track the lane, which can greatly improve the ability of stability and anti-jamming for the system. Therefore, the information of road structure model is combined with extended Kalman filter. The extended Kalman filter is used to track after the lane lines were detected, which greatly improved the accuracy of lane identification. The effectiveness and robustness of the algorithm are ensured too.

\subsection{Lane Model}

The lane model is built according to the roadway geometry and vehicle dynamics (Dickmanns et al., 1992, Watanabe et al., 2009). The road boundary model is defined as follows:

$$
x_{t, k}(z)=\frac{1}{2} k W_{t}+e_{t}+\theta_{t} z+\frac{1}{2} c_{0, t} z^{2}+\frac{1}{6} c_{1, t} z^{3}
$$

$x_{t, k}(z)$ is the position for the point of lane boundary which has a distance of $z$ from the vehicle at time $t$. $k$ denotes the left or right side of the lane with $k=-1$ or $k=1$. $e_{t}$ is the lateral offset between the center of the vehicle and the center of the lane. $\theta_{t}$ is the yaw angle between driving direction and the lane. $c_{0, t}, c_{1, t}$ are the curvature and the change rate of curvature. $\phi_{t}$ is the pitch angle between the optical axis and the plane of the road plane. The estimated parameters are described as follows:

$$
x(t)=\left(\dot{\theta}_{t}, \theta_{t}, \dot{e}_{t}, e_{t}, c_{1, t}, c_{0, t}, \phi_{t}, W_{t}\right)
$$

The '-' on the letters in the formula expresses the change rate of corresponding parameters. Compared with the models (Dickmanns et al., 1992, Watanabe et al., 2009), the model in this paper increases two new estimated parameters, that is, the change rate of the yaw angle $\dot{\theta}_{t}$ and the lateral offset $\dot{e}_{t}$. The increase of the track estimation parameters of the lane information can correspondingly improve the stability, anti-interference and detection rate of the lane detection system..

\subsection{Extended Kalman Filtering and Parameter Estimation}

When the candidate lanes have been detected preliminarily, they are tracked and estimated by Kalman filter. The traditional Kalman filter is useful when the state equation and measurement equation of system are both linear system. The system noise and measurement noise are consistent with the Gaussian distribution (Xiao et al., 2018). The parameters can be estimated by the principle of minimum mean square error. But the state equation and measurement equation of the lane model in this paper are nonlinear. The traditional Kalman filter is not practicable in this system. So, the nonlinear system should be approximated by a linear problem. The Taylor series expansion is the most commonly used linearization method, which ignores the higher order term. So the Kalman filter is replaced by the extended Kalman filtering (EKF)(Welch et al., 2001). The update processing of extended Kalman filter is shown as following. 
The process has a state vector $x \in R^{n}$. It is now governed by the non-linear stochastic difference equation:

$$
\left\{\begin{array}{l}
x(t)=f(x(t-1), u(t-1), w(t-1)) \\
y(t)=h(x(t), v(t))
\end{array}\right.
$$

where the random variables $w(\cdot), v(\cdot)$ represent the process and measurement noise with zero-mean. The $u(t-1)$ is the driving function. The time update of state vector and the observation vector for extended Kalman filter are as following:

$$
\left\{\begin{array}{l}
\hat{x}_{p}(t)=f(\hat{x}(t-1), u(t-1), 0) \\
P_{p}(t)=A(t) P(t-1) A(t)^{T}+W(t) Q(t-1) W(t)^{T}
\end{array}\right.
$$

$\hat{x}_{p}(t)$ is the priori state estimate at time $t$ where $\hat{x}(t)$ is the posteriori state estimate. $A(t)$ and $W(t)$ are the process Jacobians at time $t$. Where $P_{p}(t)$ is the priori estimate error covariance, $P(t)$ is the posteriori estimate error covariance. $Q(t)$ is the process noise covariance. The Kalman gain in the update of measurement is shown as following:

$$
K(t)=\left(P_{p}(t) H(t)^{T}\right)\left(H(t) P_{p}(t) H(t)^{T}+V(t) R(t) V(t)^{T}\right)^{-1}
$$

where $H(t)$ and $V(t)$ are the measurement Jacobians at time $t . R(t)$ is the measurement noise covariance. The covariance matrix of posteriori estimation error in the measurement update is shown as:

$$
P(t)=P_{p}(t)-K(t) H(t) P_{p}(t)
$$

The updated estimation of observation vector in the measurement update is the following expression:

$$
\hat{x}(t)=\hat{x}_{p}(t)+K(t)\left(y(t)-h\left(\hat{x}_{p}(t), 0\right)\right)
$$

According to the change of traffic variables and vehicle dynamics, system matrix can be obtained as follows (Dickmanns et al., 1992): 


$$
A(t)=\left(\begin{array}{cccccccc}
1 & 0 & 0 & 0 & 0 & 0 & 0 & 0 \\
\Delta t & 1 & 0 & 0 & 0.5 \cdot \Delta t^{2} \cdot V^{2} & 0 & 0 & 0 \\
0 & 0 & 1 & 0 & \Delta t^{2} \cdot V^{3} & 0 & 0 & 0 \\
0 & 0 & \Delta t & 1 & 0 & 0 & 0 & 0 \\
0 & 0 & 0 & 0 & 1 & 0 & 0 & 0 \\
0 & 0 & 0 & 0 & \Delta t \cdot V & 1 & 0 & 0 \\
0 & 0 & 0 & 0 & 0 & 0 & 1 & 0 \\
0 & 0 & 0 & 0 & 0 & 0 & 0 & 1
\end{array}\right)
$$

where $V$ is the vehicle speed, the observation vector is set as $x_{\text {road }}$. Because the estimation points which are selected to estimate parameters are image pixels, the points need to be converted:

$\left\{\begin{array}{l}y_{\text {img }}=-f \cdot h / z_{\text {road }}-f \cdot \phi_{t} \\ x_{\text {img }}=f \cdot x_{\text {road }} / z_{\text {road }}\end{array}\right.$

In keeping with road plane, $x_{i m g}$ is image's ordinate and $y_{i m g}$ is abscissa. The jacobian matrix for the partial derivatives of $h$ respect to $x$ is defined as follows:

$$
H_{[i, j]}=\frac{\partial h_{[i]}}{\partial x_{[j]}}\left(\tilde{x}_{k}, 0\right)=\left(\frac{\partial x_{i m g}}{\partial x[0]}, \frac{\partial x_{i m g}}{\partial x[1]}, \frac{\partial x_{i m g}}{\partial x[2]}, \cdots, \frac{\partial x_{i m g}}{\partial x[7]}\right)
$$

So, from (16) there are:

$$
\left\{\begin{array}{l}
\frac{\partial x_{i m g}}{\partial x[i]}=\frac{f}{z_{\text {road }}} \cdot \frac{\partial x_{\text {road }}}{\partial x[i]}, \quad i=0,1, \ldots, 5,7 \\
\frac{\partial x_{\text {img }}}{\partial x[6]}=\frac{\partial x_{\text {img }}}{\partial z_{\text {road }}} \cdot \frac{\partial z_{\text {road }}}{\partial x[6]}
\end{array}\right.
$$

From (8), we can get that:

$$
x_{\text {road }}=\frac{1}{2} k W_{t}+e_{t}+\theta_{t} z_{\text {road }}+\frac{1}{2} c_{0, t} z_{\text {road }}^{2}+\frac{1}{6} c_{1, t} z_{\text {road }}^{3}
$$

And from (9), we obtain that:

$$
\left(\frac{\partial x_{\text {road }}}{\partial x[0]}, \frac{\partial x_{\text {road }}}{\partial x[1]}, \cdots, \frac{\partial x_{\text {road }}}{\partial x[7]}=\left(0, z_{\text {road }}, 0,1, \frac{1}{6} z_{\text {road }}^{3}, \frac{1}{2} z_{\text {road }}^{2}, 0, \frac{1}{2} k\right)\right)
$$


Since there is no $\phi_{t}$ in (19), $x[6]$ can be computed by:

$\frac{\partial x_{i m g}}{\partial x[6]}=\frac{\partial x_{i m g}}{\partial z_{\text {road }}} \cdot \frac{\partial z_{\text {road }}}{\partial x[6]}$

According to the relationship between angle and ordinate in image, from (9) (16) it can be gotten that:

$\frac{\partial x_{r o a d}}{\partial x[6]}=\frac{f^{2} \cdot h}{\left(y_{i m g}+f \cdot \phi_{t}\right)^{2}}$

Note the definition of $x(t)$ in (9), it can be obtained:

$\frac{\partial x_{\text {road }}}{\partial z_{\text {road }}}=\theta_{t}+c_{0, t} z_{\text {road }}+\frac{1}{2} c_{1, t} z_{\text {road }}^{2}$

So, from (16) (19) (23) we have:

$\frac{\partial x_{\text {img }}}{\partial z_{\text {road }}}=\frac{\partial x_{\text {img }}}{\partial x_{\text {road }}} \frac{\partial x_{\text {road }}}{\partial z_{\text {road }}}=f \cdot\left(\frac{1}{2} c_{0, t}+\frac{1}{3} c_{1, t} z_{\text {road }}-\frac{k W_{t}}{2 z_{\text {road }}^{2}}-\frac{e_{t}}{z_{\text {road }}^{2}}\right)$

Then:

$H_{[i, j]}=\left(0, f, 0, \frac{f}{z_{\text {road }}}, \frac{f \cdot z_{\text {road }}^{2}}{6}, \frac{f \cdot z_{\text {road }}}{2}, \frac{\partial x_{\text {img }}}{\partial z_{\text {road }}} \frac{\partial z_{\text {road }}}{\partial x[6]}, \frac{k \cdot f}{2 \cdot z_{\text {road }}}\right)$

The 7th item in the Jacobian matrix $H$ can be obtained by the above formula. The parameters of lane model can be expressed by the state vector and the covariance matrix of estimation error. The abscissas of the lane boundary points in the original input image selected from the above steps, are gotten by the extended Kalman filter to conduct iterative estimation. If the extracted lane candidate boundary points include the noise introduced by observation, but not include wrong identification points, then the unit of standard deviation for noise is pixel. If the value of the variance for estimation error is $\sigma^{2}$, then the maximum change of each parameter is $3 \sigma$. Assuming that the standard deviation of $x_{i m g}$ is $\sigma$, the search area of candidate points is $\left(x_{i m g}-3 \sigma, y_{i m g}\right) \sim\left(x_{i m g}+3 \sigma, y_{i m g}\right)$ for the next time. The selection of lane curvature also can be narrowed down too.

\section{EXPERIMENTS AND ANALYSIS}

The numerous experiments were implemented to verify the validity of the proposed algorithm with the programming development environment VS2010 on the platform of Intel Core i5-6500 processor at $3.2 \mathrm{GHz} 8 \mathrm{~GB}$ cache. The operating system is Windows 7. The algorithm is based on monocular 
Table 1. The detection efficiency

\begin{tabular}{|l|l|l|l|l|l|l|l|}
\hline \multirow{2}{*}{$\begin{array}{l}\text { Number } \\
\text { of Video }\end{array}$} & \multirow{2}{*}{$\begin{array}{c}\text { Total } \\
\text { Frames }\end{array}$} & \multicolumn{3}{|c|}{ Correctly Detected Frames } & \multicolumn{3}{c|}{ Recognition Rate } \\
\cline { 3 - 8 } & & Proposed & Xiao (2015) & Aly (2008) & Proposed & Xiao (2015) & Aly (2008) \\
\hline 1 & 1805 & 1691 & 1608 & 1651 & $93.7 \%$ & $89.1 \%$ & $91.5 \%$ \\
\hline 2 & 1377 & 1283 & 1250 & 1246 & $93.2 \%$ & $90.8 \%$ & $90.5 \%$ \\
\hline 3 & 2222 & 2102 & 2033 & 2070 & $94.6 \%$ & $91.5 \%$ & $93.2 \%$ \\
\hline 4 & 1286 & 1196 & 1138 & 1157 & $93.0 \%$ & $88.5 \%$ & $90.0 \%$ \\
\hline
\end{tabular}

vision. The focal length is $8 \mathrm{~mm}$. The maximum frame rate of image is 30 frames per second. The size of image is $1280 \times 720$ in pixels. Experiments show that proposed algorithm takes less than $34 \mathrm{~ms}$ to process a frame of picture.

To verify the effectiveness and robustness of the proposed algorithm, the road video data captured from Wuhan is selected to test. These data include a variety of road conditions, such as trees shadow, pedestrian, vehicle interference, strong light and shadow, curve and so on. Test results of four video sequences are shown as example. It is difficult to find open source code on the Internet because of the complexity of lane line detection algorithm engineering. So, the comparison algorithms are RANSAC algorithm based on template (Aly, 2008) and our previously proposed algorithm based on Beamlet Transformation and K-means Clustering (Xiao et al., 2015). Table 1 is the statistics result for the lane detection, which include the total number of the test frames and the recognition rate.

From the data in Table 1, the recognition rate of the proposed algorithm in this paper is $2 \%$ to $3 \%$ higher than the RANSAC algorithm and is 3\% to 5\% higher than the Beamlet and K-means algorithm. The algorithm has good stability and high recognition rate up to $94.6 \%$. The proposed algorithm in this paper has good accuracy and robustness for the structural road environment. According to the geometry of road and the moving of vehicle, a new driveway model is set up. The evaluated parameters of driveway information are increased. At the same time, the lane inner boundary is obtained using the various constraints of the lane in order to eliminate interference lines. The coordinate parameters of lane edge points are tracked and estimated by the extended Kalman filter, which can ensure the stability and robustness of the algorithm. Hence, the detection result is very stable and the problems of jitter and low fitting precision in traditional algorithm are missed.

Next, it is necessary to discuss the robustness of the lane detection algorithm under different road environment. The comparative analysis of lane detection effect for complex road environment is shown in Figures 7-9. It includes lane-changing, curve, character interference, strong light and shadow trees, vehicles, and so forth.

In Figure 7, the RANSAC algorithm and the Beamlet and K-means algorithm have mistaken identification during changing lanes, while the proposed algorithm solves the problem well in Figure 7, bottom. The RANSAC algorithm and the Beamlet and K-means algorithm have the problem of instability and inaccuracy fitting in curve lane in Figure 8, while the proposed algorithm still has high stability and robustness. In the case of closing to the character interference in Figure 9, the RANSAC algorithm and the Beamlet and K-means algorithm make a mistake in detection, but the proposed algorithm has a better result. In Figure 10 top, the length of left lane detected by RANSAC algorithm is greatly reduced because of the vehicle interference and the detection result of the Beamlet and $\mathrm{K}$-means algorithm is not accurate, while the detection result of the proposed algorithm is still very accurate in Figure 10, bottom, although there are lots of mistaken edge points. With the strong light in Figure 11, the RANSAC algorithm detected the right lane only, and the Beamlet and K-means algorithm's detection results are not accurate, while both lanes are detected by the proposed algorithm. In Figure 12, the RANSAC algorithm can detect the left lane correctly which has less shadow, but 
Figure 7. Detection results for lane-changing. Top to bottom: Aly(2008), Xiao(2015), proposed.
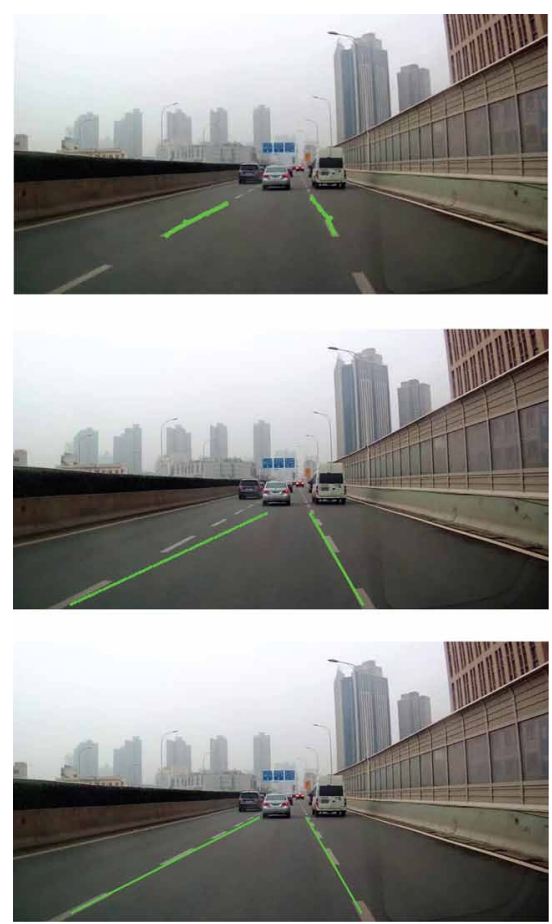

Figure 8. Detection results for curve. Top to bottom: Aly(2008), Xiao(2015), proposed.
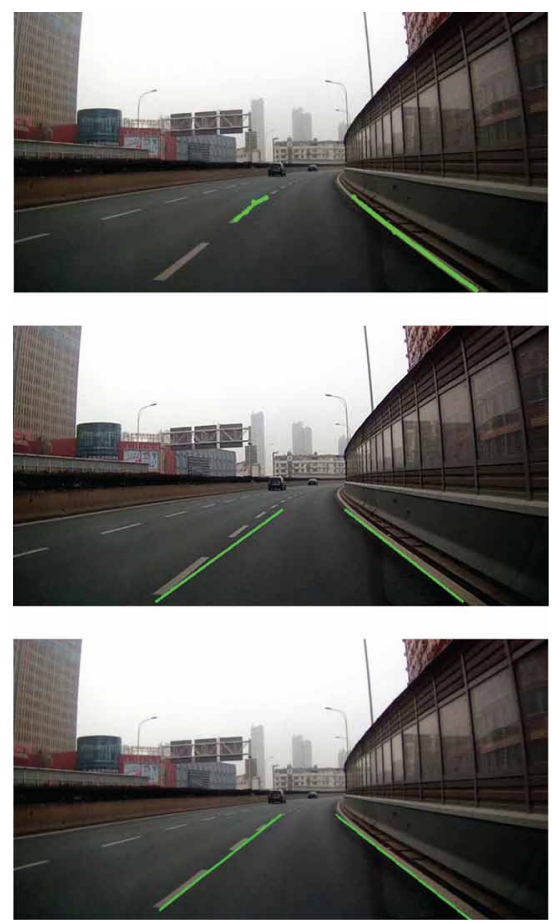
Figure 9. Detection results for character interference. Top to bottom: Aly (2008), Xiao (2015), proposed.
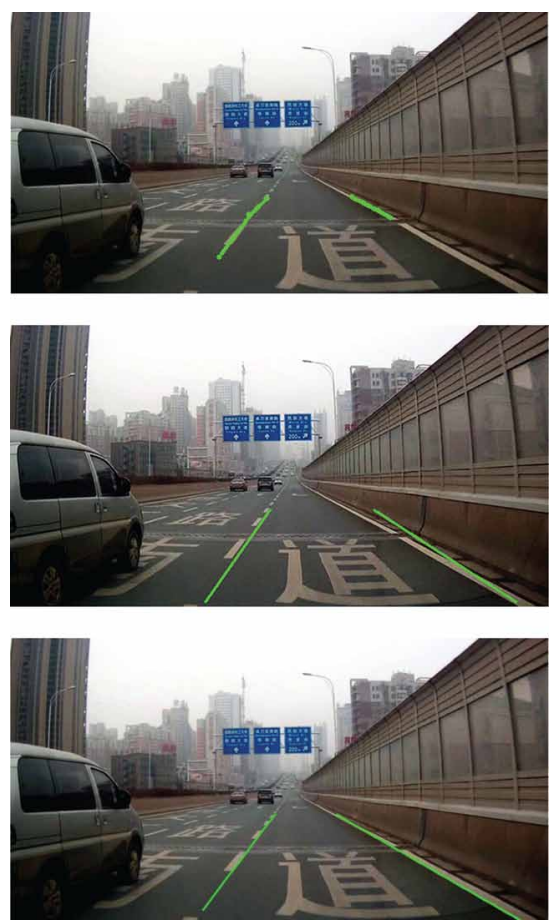

it is unable to identify right lane with serious shadow. And the results of the Beamlet and K-means algorithm are similar to it. The proposed algorithm can accurately detect it with high robustness.

From the comparison of detection results above in various complex road conditions, such as lane-changing, curve, the zebra crossing, strong light, shadow trees and vehicles, etc. The efficient and stability of the proposed algorithm are higher than the RANSAC algorithm and the Beamlet and $\mathrm{K}$-means algorithm. The proposed algorithm in this paper can accurately identify the lane for various special road conditions. At the same time, it can achieve real-time processing speed (30 frames per second), which has higher recognition rate and reliability compared with RANSAC algorithm and Beamlet and K-means algorithm. Figures 13 and 14 show the detection results of dashed line and double line. Of course, the proposed algorithm also has some shortcomings.

For example, in Figure 15, when the vehicle changes the lane and there are long interference lines which are parallel to the lanes at the same time (Figure 15, top), there will be a mistaken detection (Figure 15, bottom). Further research will be needed later.

\section{CONCLUSION}

A new lane detection algorithm based on monocular vision was proposed in this paper. The road structure and vehicle dynamics are used to set up a new driveway model, where the parameters of driveway information to be evaluated are increased. A suitable custom parameter space is established in the proposed algorithm. The traditional Hough transform is improved in the proposed algorithm to increase the processing speed. The combination of lane model with extended Kalman filter for the lane detection guarantees effectively the stability of the algorithm. The problem of instability and the accuracy for lane fitting is improved here too. Experiments show that the algorithm has good recognition rate and robustness in various complex road environments. The algorithm did not give accurate confidence level of lane detection. It will be further studied in the follow-up work. 
Figure 10. Detection results for vehicle interference. Top to bottom: Aly (2008), Xiao (2015), proposed.
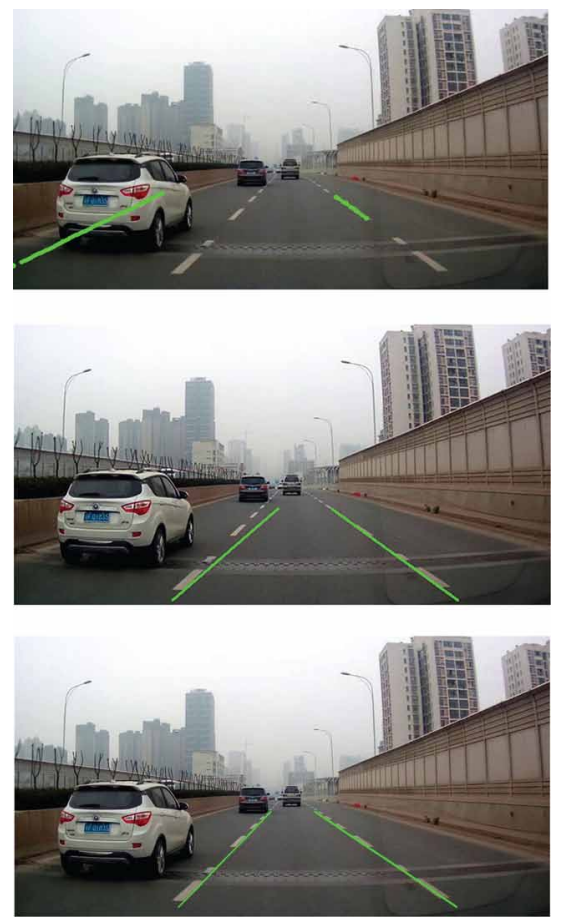

Figure 11. Detection results for strong light. Top to bottom: Aly (2008), Xiao (2015), proposed.
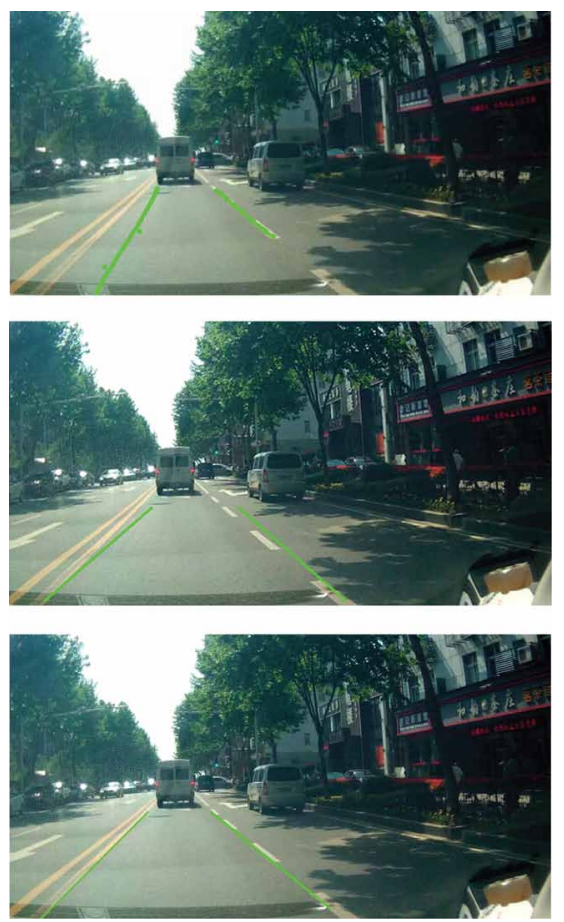
International Journal of Digital Crime and Forensics

Volume $12 \cdot$ Issue $2 \cdot$ April-June 2020

Figure 12. Detection results for shadow. Top to bottom: Aly (2008), Xiao (2015), proposed.
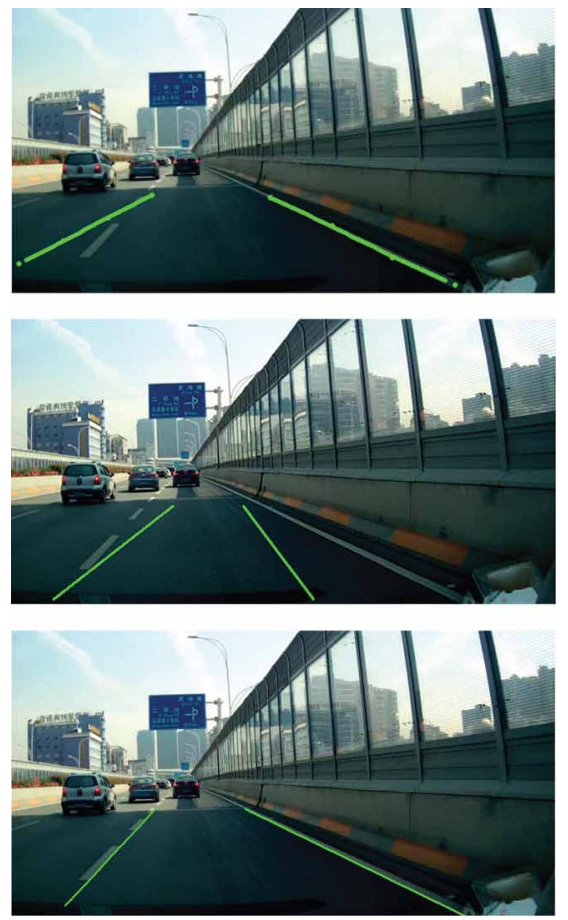

Figure 13. Detection results for dashed line
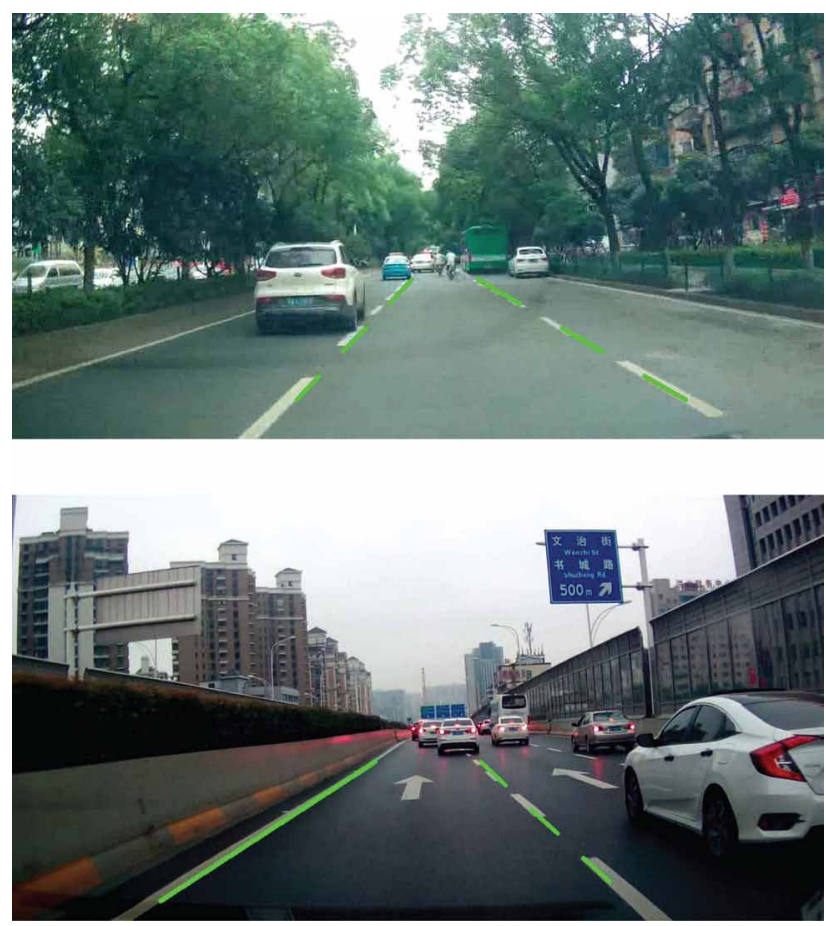
Figure 14. Detection result of double line
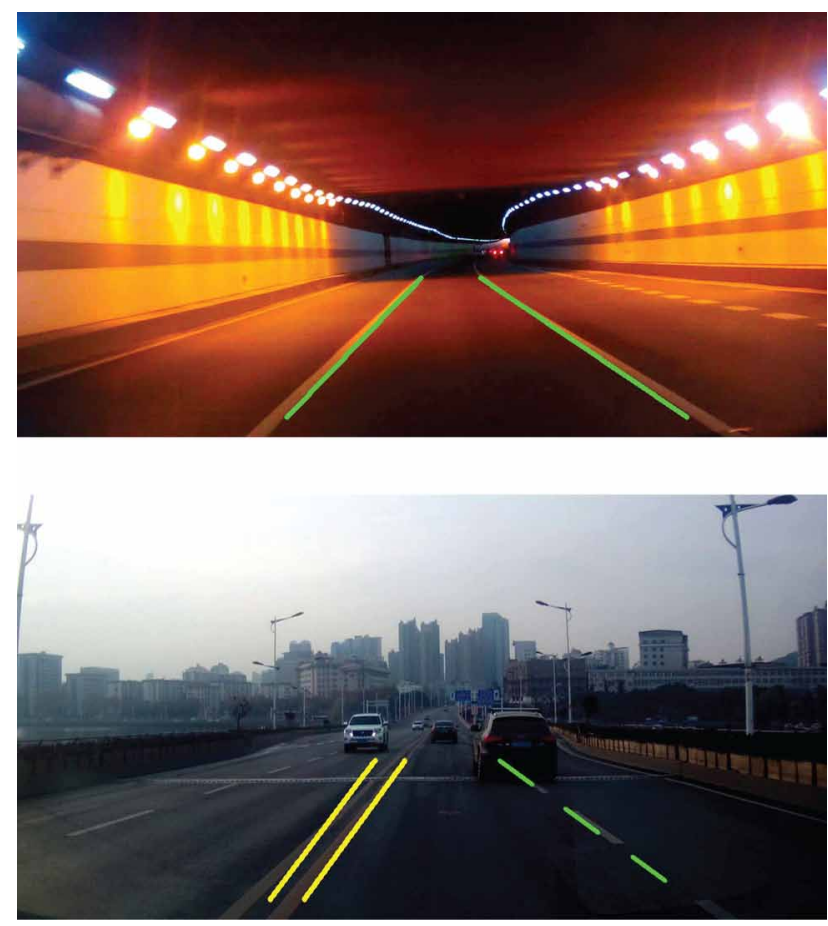

Figure 15. False detection results for the proposed method
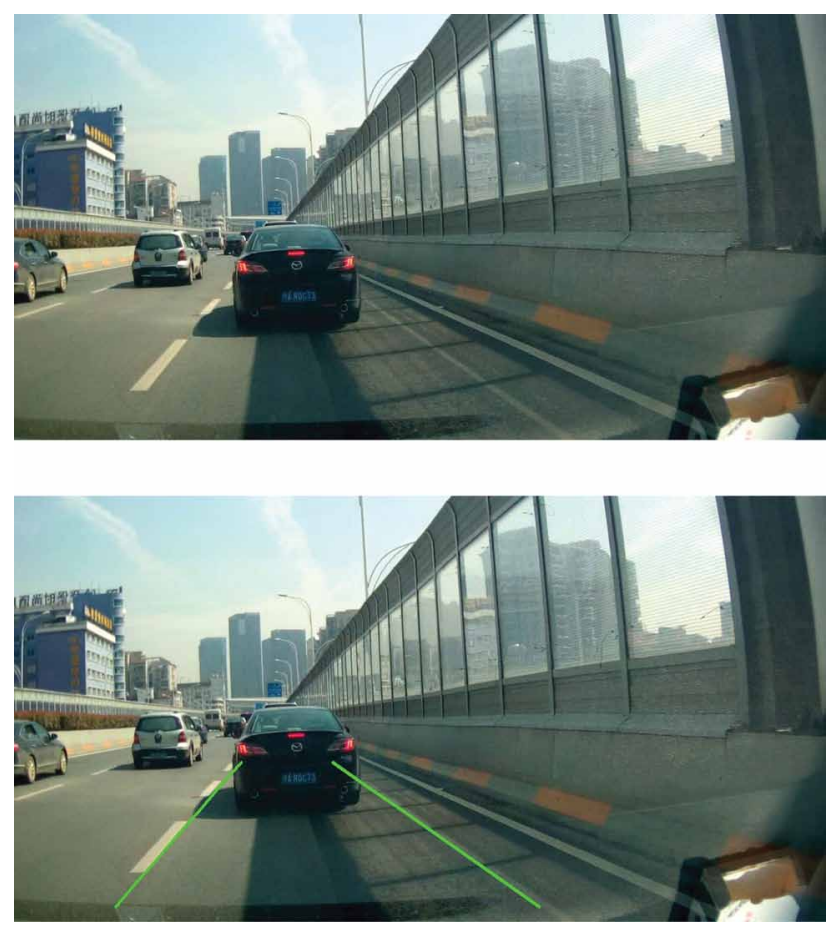
International Journal of Digital Crime and Forensics

Volume $12 \cdot$ Issue $2 \cdot$ April-June 2020

\section{ACKNOWLEDGMENT}

This research was supported by National Natural Science Foundation of China [Grant No. 61471272];

Natural Science Foundation of Hubei Province, China [Grant No. 2016CFB499]. 


\section{REFERENCES}

Aly, M. (2008). Real time detection of lane markers in urban streets. In Proceedings of the IEEE Intelligent Vehicles Symposium (pp. 7-12). IEEE. doi:10.1109/IVS.2008.4621152

Dickmanns, E. D., \& Mysliwetz, B. D. (1992). Recursive 3-D Road and Relative Ego-State Recognition. IEEE Transactions on Pattern Analysis and Machine Intelligence, 14(2), 199-213. doi:10.1109/34.121789

Hillel, A. B., Lerner, R., Dan, L., \& Raz, G. (2014). Recent progress in road and lane detection: A survey. Machine Vision and Applications, 25(3), 727-745. doi:10.1007/s00138-011-0404-2

Hough, P. V. (1962). U.S. Patent No. 3,069,654. Washington, DC: U.S. Patent and Trademark Office.

Huang, A. S., Moore, D., Antone, M., Olson, E., \& Teller, S. (2009). Finding multiple lanes in urban road networks with vision and lidar. Autonomous Robots, 26(2-3), 103-122. doi:10.1007/s10514-009-9113-3

Kazui, M., Haseyama, M., \& Kitajima, H. (2010). Estimation of the vanishing point for automatic driving system using a cross ratio. Systems and Computers in Japan, 33(9), 31-40. doi:10.1002/scj.10090

Kortli, Y., Marzougui, M., \& Atri, M. (2017). Efficient implementation of a real-time lane departure warning system (pp. 1-6). IEEE Image Processing, Applications and Systems.

Lee, D. K., Shin, J. S., Jung, J. H., Park, S. J., Oh, S. J., \& Lee, I. S. (2017). Real-time lane detection and tracking system using simple filter and Kalman filter. In Proceedings of the IEEE 9th International Conference on Ubiquitous and Future Networks (pp. 275-277). IEEE.

Lee, J. W. (2002). A machine vision system for lane-departure detection. Computer Vision and Image Understanding, 86(1), 52-78. doi:10.1006/cviu.2002.0958

McDonald, J., Franz, J., \& Shorten, R. (2001). Application of the Hough Transform to lane detection in Motorway Driving Scenarios. In Proceedings of the Irish Signals and Systems Conference. Academic Press.

Mechat, N., Saadia, N., M'Sirdi, N. K., \& Djelal, N. (2013). Lane detection and tracking by monocular vision system in road vehicle. In Proceedings of the IEEE International Congress on Image and Signal Processing (pp. 1276-1282). IEEE.

Pan, X., Si, W., \& Ogai, H. (2017). Fast Road Detection Based on a Dual-Stage Structure. In Proceedings of 9th International Conference on Computer and Automation Engineering (pp. 155-162). Academic Press. doi:10.1145/3057039.3057101

Li, Q., Zhou, J., Li, B., Guo, Y., \& Xiao, J. (2018). Robust lane-detection method for low-speed environments. Sensors (Basel), 18(12), 4274. doi:10.3390/s18124274

Shin, B. S., Tao, J., \& Klette, R. (2015). A super particle filter for lane detection. Pattern Recognition, 48(11), 3333-3345. doi:10.1016/j.patcog.2014.10.011

Wang, Y., Teoh, E. K., \& Shen, D. (2004). Lane detection and tracking using b-snake. Image and Vision Computing, 22(4), 269-280. doi:10.1016/j.imavis.2003.10.003

Watanabe, A., Naito, T., \& Ninomiya, Y. (2009). Lane detection with roadside structure using on-board monocular camera. In Proceedings of the IEEE Intelligent Vehicles Symposium (pp. 191-196). IEEE. doi:10.1109/ IVS.2009.5164276

Welch, G., \& Bishop, G. (2001). An Introduction to the Kalman Filter. University of North Carolina at Chapel Hill.

Xiao, J., Chen, X., Li, B., Gao, W., \& Peng, H. (2015). Lane detection algorithm based on beamlet transformation and k-means clustering. Journal of Sichuan University, 47(4), 98-103.

Xiao, J., Tian, H., Zhang, Y., Zhou, Y., \& Lei, J. (2018). Blind video denoising via texture-aware noise estimation. Computer Vision and Image Understanding, 169, 1-13. doi:10.1016/j.cviu.2017.11.012

Xu, H. R., Wang, X. D., \& Fang, Q. (2011). Structure road detection algorithm based on b-spline curve model. Acta Automatica Sinica, 37(3), 270-275. doi:10.3724/SP.J.1004.2011.00270 
Xu, Z., Shin, B. S., \& Klette, R. (2015). Closed form line-segment extraction using the Hough transform. Pattern Recognition, 48(12), 4012-4023. doi:10.1016/j.patcog.2015.06.008

Jinsheng Xiao received a Doctoral degree in Mathematics from Wuhan University, China, in 2001. From 2001 to 2004, he was a Research Assistant with the Institute of Multimedia Network Communication, Wuhan University. From 2004 to 2008, he was Lecturer with School of Electronic Information, Wuhan University. From Dec 2008 to present he is an Associate Professor for Information and Communication Engineering with School of Electronic Information. Between Aug. 2014 and Aug. 2015, he was a Visiting Scholar with University of California, Santa Barbara, USA. He has authored or co-authored more than 50 scientific articles in journals, books, and conference proceedings. His research interests include video and image processing, computer vision, and multimedia network communication.

Liang Li was born in Wuhu City, China in 1995. He received the B.E. degree in Electronic Information Engineering from Wuhan University of Technology, China, in 2018. He is currently a postgraduate student in signal and information processing at Wuhan University. His research interests include video processing, and computer vision.

Yuan Yao received his B.S. in Electronic technology and his M.S. degree in control theory and control system form Wuhan University of Technology, China, in 1997 and 2002, respectively. He received his PhD in communication and information systems from Wuhan University, China in 2008. From 2002 to 2008. In2012, He joined in College of Physical Science and Technology, Central China Normal University, China as an associate professor. Currently, his research interests focus on signal processing.

Wenxin Xiong was born in Wuhan City, China in 1998. She received the B.E. degree in Electrical Engineering and automation from Wuhan University, China, in 2019. She is currently a postgraduate student in Electronic and communication engineering at Wuhan University. Her research interests include image processing and computer vision.

Reinhard Klette (Auckland University of Technology, Fellow of the Royal Society of New Zealand) made significant contributions to two major areas, digital geometry and computer vision. Professor Klette has been working in the area of computer vision for more than 30 years. He is the director of the Centre for Robotics \& Vision (CeRV) of Auckland University of Technology. 